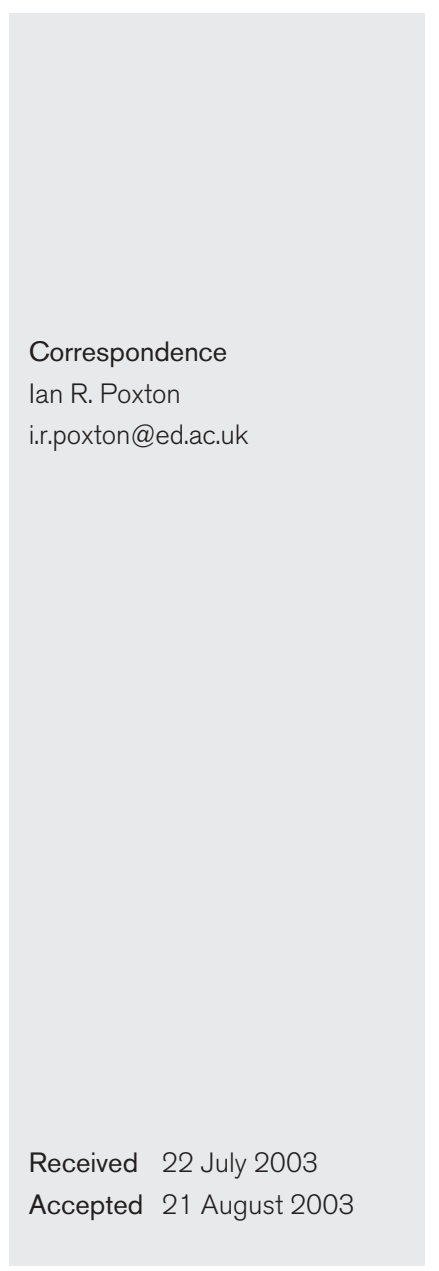

\title{
Effects of sub-MIC concentrations of antibiotics on growth of and toxin production by Clostridium difficile
}

\author{
Lisa J. Drummond, David G. E. Smith and lan R. Poxton \\ Medical Microbiology, Centre for Infectious Diseases, The University of Edinburgh Medical School, \\ Teviot Place, Edinburgh EH8 9AG, UK
}

\begin{abstract}
Effects on growth and toxin A production of sub-MIC concentrations of six different antibiotics were investigated in three strains of Clostridium difficile: reference strain NCTC 11223, a fully sequenced strain (630) and a locally endemic isolate (strain 338a). The antibiotics chosen for investigation were the agents used to treat $C$. difficile-associated disease (CDAD), i.e. vancomycin and metronidazole, and four antibiotics that are commonly involved in precipitating CDAD (amoxycillin, clindamycin, cefoxitin and ceftriaxone). Strains were cultured in sublethal concentrations of antibiotics (1/2, $1 / 4$ and 1/8 MIC) over $104 \mathrm{~h}$ and growth and toxin A production were measured three times a day. Effects varied between strain and antibiotic. The most common effect on growth of the strains was to increase the initial lag period by approximately $4 \mathrm{~h}$, compared with antibiotic-free controls; however, strain NCTC 11223, which has high-level clindamycin resistance ( $\left.\geqslant 512 \mu \mathrm{g} \mathrm{ml}^{-1}\right)$, showed no lag whatsoever in comparison with the controls when grown in this antibiotic. The most common effect on production of toxin A was in the onset of toxin elaboration. Normally, toxins began to appear at low levels in the early stationary phase, before accumulating to high levels by the start of decline. In the presence of sub-MIC antibiotics, this onset appeared before that of the antibiotic-free controls. This effect was seen with metronidazole, amoxycillin and clindamycin, rarely with vancomycin and never with cefoxitin. These results suggest a very complex, strain-dependent relationship between the effects of growth and toxin production.
\end{abstract}

\section{INTRODUCTION}

Clostridium difficile is the most common cause of antibioticassociated diarrhoea and is the aetiological agent of pseudomembranous colitis. This Gram-positive, obligately anaerobic spore-former causes a wide spectrum of disease, ranging from mild, self-limiting diarrhoea to serious diarrhoea and, in some cases, complications such as pseudomembrane formation, toxic megacolon and peritonitis. The main bacterial factors that are recognized in $C$. difficileassociated disease (CDAD) are two high-molecular-mass toxins, $\mathrm{A}$ and $\mathrm{B}$. Once released onto the gut mucosa, they act in concert to produce the characteristic pathology and symptoms. Antibiotics have a prominent role in C. difficile disease: it is hypothesized that depletion of bowel microbiota by antibiotics leads to the elimination of any resistance to colonization by $C$. difficile (Farrell \& LaMont, 2000). Broadspectrum agents, such as clindamycin, amoxycillin and third-generation cephalosporins, are associated with the greatest risk of developing $C$. difficile diarrhoea, although almost all antibiotics have been implicated at one time or another (Mylonakis et al., 2001).

Abbreviation: CDAD, Clostridium difficile-associated disease.
Toxin production is affected by environmental factors that include temperature, glucose concentration, biotin limitation and amino acid concentration (Onderdonk et al., 1979; Honda et al., 1983; Barc et al., 1992; Yamakawa et al., 1996; Dupuy \& Sonenshein, 1998; Ikeda et al., 1998; Karlsson et al., 1999). Studies have shown that subinhibitory levels of antibiotics have an effect on the production of toxin: Onderdonk et al. (1979) found that subinhibitory concentrations of vancomycin and penicillin increased toxin production by C. difficile and Honda et al. (1983) found that clindamycin and cephaloridine increased the production of toxin A. Other antibiotics may also affect this process. The aim of this study was to investigate and clarify the effect of antibiotics on growth and production of toxin A by $C$. difficile. Six different antibiotics -including agents that either precipitate CDAD or are used for its treatment - and three different strains of $C$. difficile were investigated.

\section{METHODS}

Strains of $\boldsymbol{C}$. difficile. Three strains of $C$. difficile were used in this study: NCTC 11223, strain 630 (fully sequenced) and strain 338a (a local endemic strain that was collected during a recent epidemiology study). 
Strain 338a is of S-type 5236 (ribotype 1) and was present in $78 \%$ of cases of $C$. difficile diarrhoea in a geriatric unit in the Royal Victoria Hospital, Edinburgh (McCoubrey et al., 2003). Strains were grown from spores stored in cooked meat broth [anaerobic investigation medium (AIM) with cooked meat particles (Brown et al., 1996)]. A loopful (approx. $30 \mu \mathrm{l}$ ) was added to $3 \mathrm{ml}$ pre-reduced AIM and incubated anaerobically overnight $\left(80 \% \mathrm{H}_{2}, 10 \% \mathrm{~N}_{2}, 10 \% \mathrm{CO}_{2}\right.$ at $\left.37^{\circ} \mathrm{C}\right)$ to yield approximately $10^{8}$ cells $\mathrm{ml}^{-1}$. Appropriate purity checks were carried out on starter cultures before use.

Antibiotics. Antibiotics chosen for the study were vancomycin (V2002; Sigma) and metronidazole (M1547; Sigma), two agents that are used for the treatment of CDAD, and four agents that are associated with precipitation of the disease: amoxycillin (A8523; Sigma); clindamycin (C5269; Sigma); cefoxitin (C4786; Sigma) and ceftriaxone (C5793; Sigma). Second-generation cephalosporins (including cefoxitin) are less commonly associated with $C$. difficile disease, although they still possess good anti-anaerobe activity. Cefoxitin, present in the selective medium at $8 \mathrm{mg} \mathrm{l}^{-1}$, was chosen for comparison with the thirdgeneration cephalosporin ceftriaxone. MICs of these strains to the six antibiotics were determined by broth macrodilution (National Committee for Clinical Laboratory Standards, 1997) and are shown in Table 1. Concentrations of antibiotics used in this study corresponded to $1 / 2$, $1 / 4$ and $1 / 8$ of the MIC, except in the case of clindamycin with strain NCTC 11223. This strain was highly resistant and $512 \mu \mathrm{g} \mathrm{ml}^{-1}$, the highest concentration achievable in the study, allowed growth of this strain. Antibiotics were prepared in sterile distilled water as $100 \times$ solutions with reference to the highest concentration required. Doubling dilutions were made in sterile distilled water and 1 vol. antibiotic was added to 100 vols broth.

Growth curves and toxin production. Preliminary growth curves were determined to relate optical density $\left(\mathrm{OD}_{600}\right)$ to viable counts and toxin A production (by semi-quantitative analysis - see below) over a period of $104 \mathrm{~h}$. To ensure that three sub-MIC concentrations of antibiotics were used for each strain, four additional concentrations of antibiotic were used, two above and two below the predicted MIC, to allow for any minor differences in MIC. Where necessary, the extra determinations were discarded retrospectively. An antibiotic-free control was used for each strain for comparison. An inoculum of $10^{6}$ cells $\mathrm{ml}^{-1}$ was used and growth $\left(\mathrm{OD}_{600}\right)$ was measured three times a day at 0 , 4 and $8 \mathrm{~h}$ for 5 days. A $1 \mathrm{ml}$ sample was removed for OD measurement. After measuring the OD, the sample was transferred to a $3 \mathrm{ml}$ Eppendorf tube, centrifuged for $2 \mathrm{~min}$ at $13000 \mathrm{~g}$ and the supernate was transferred to a fresh Eppendorf tube and stored at $-20^{\circ} \mathrm{C}$ for toxin analysis.

Toxin A ELISA. Toxin A levels were assayed by ELISA with a ToxA kit (Techlab) according to the manufacturer's instructions. Prior to assay, $100 \mu \mathrm{l}$ of each sample was diluted in $100 \mu \mathrm{l}$ of the buffer provided; $100 \mu \mathrm{l}$ of this dilution was used in the assay. Plates were read at a wavelength of $450 \mathrm{~nm}$, with automatic subtraction of the $620 \mathrm{~nm}$ value. The maximum

Table 1. MICs of the six antibiotics

Abbreviations: Van, vancomycin; Met, metronidazole; Amox, amoxycillin; Clind, clindamycin; Cefo, cefoxitin; Ceft, ceftriaxone.

\begin{tabular}{|lcccrrr|}
\hline Strain & \multicolumn{6}{|c|}{ MIC $\left(\mathrm{mg} \mathrm{l}^{-1}\right)$} \\
\cline { 2 - 7 } & Van & Met & Amox & Clind & Cefo & Ceft \\
\hline NCTC 11223 & 2 & 1 & 8 & $>512$ & 256 & 64 \\
338a & 1 & 1 & 4 & 4 & 256 & 64 \\
630 & 2 & 1 & 4 & 512 & 256 & 128 \\
\hline
\end{tabular}

OD value of the assay was $3 \cdot 0$, up to which OD was linear with respect to control toxin concentration. No further dilutions of supernates were made. Results (OD values) were plotted against time to evaluate when toxin was being elaborated and to show differences between antibioticfree and -containing cultures.

\section{RESULTS AND DISCUSSION}

\section{Growth curves and toxin production}

In preliminary experiments, both viable counts and $\mathrm{OD}_{600}$ values were measured and these confirmed that over the time-period of the experiment, the values mirrored one another. Subsequently, bacterial growth was assessed by $\mathrm{OD}_{600}$ only. Fig. 1 shows growth curves and toxin levels for untreated controls of each strain. Values represent means (with standard errors) of six replicates grown on six different occasions; there was little variation in growth between strains. Each strain was clearly in the exponential phase by $8 \mathrm{~h}$ and in the stationary phase by $24 \mathrm{~h}$. Decline was then
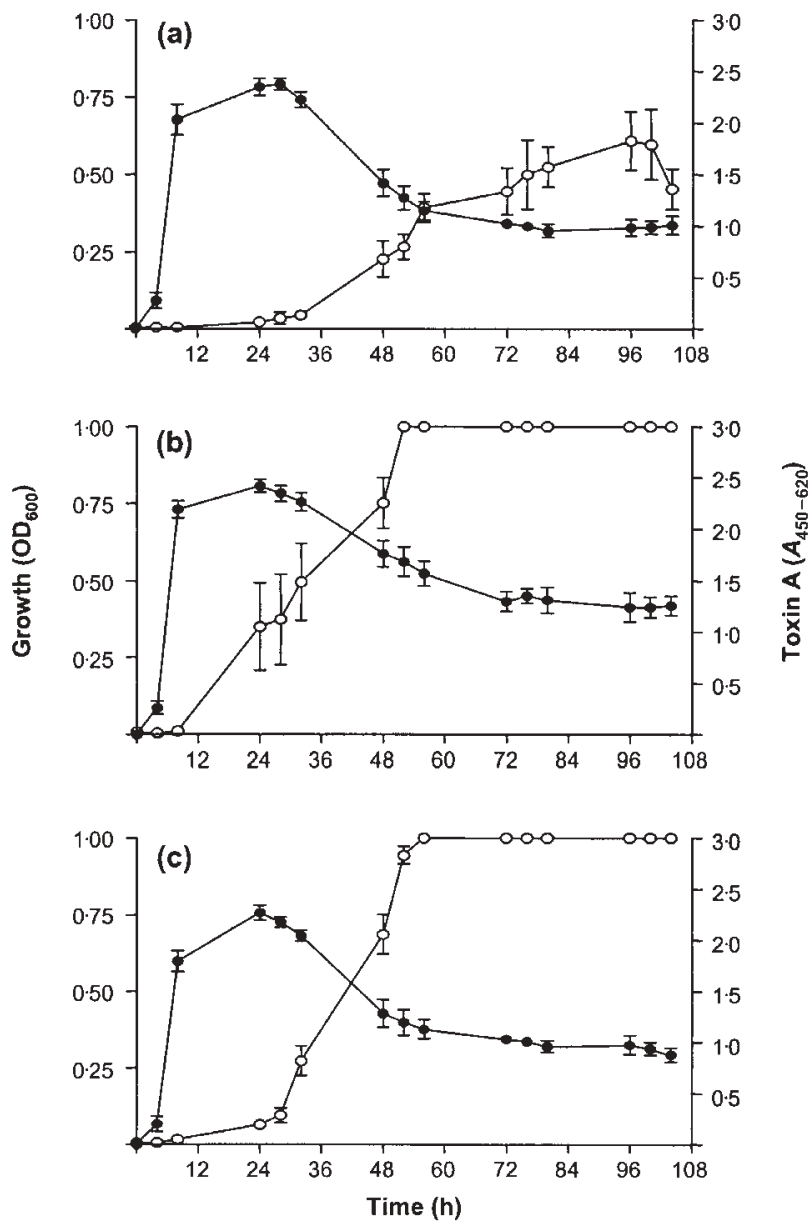

Fig. 1. Mean growth and toxin production of the strains over 104 hours: (a) strain NCTC 11223; (b) strain 338a; (c) strain 630. Growth; $\bigcirc$, toxin A production. Mean and SE were calculated from six different experiments. A reading of 3.0 is the maximum value for the toxin assay. 
apparent from $32 \mathrm{~h}$, with the OD stabilizing by approximately $56 \mathrm{~h}$. However, toxin production differed between strains, in both amount and timing of production relative to growth. Strain NCTC 11223 produced less toxin A than strains 338a and 630; toxin A production by strain NCTC 11223 rarely exceeded the measurable level of the assay $\left(\mathrm{OD}_{450}, 3 \cdot 0\right)$, whereas higher values $(>3.0)$ were obtained with strains 338a and 630. A notable difference was the point in the growth phase at which each strain produced toxin: strain 338a produced toxin during the stationary phase (by $24 \mathrm{~h}$ ), which preceded toxin production by both strains NCTC 11223 and 630 (in late stationary phase). This further correlated with the time required to produce comparable levels of toxin A, i.e. 24, 32 and $48 \mathrm{~h}$ for strains $338 \mathrm{a}, 630$ and NCTC 11223, respectively. Levels of toxin A in strains 338a and 630 generally reached maximum readable levels by approximately $52 \mathrm{~h}$.

\section{Effects of antibiotics on growth and toxin A production}

The effects of sub-MIC levels of antibiotics on bacterial growth and toxin production varied with both antibiotic and strain. Examples of the effects of antibiotics on the kinetics of growth and toxin production are shown in Fig. $2(\mathrm{a}-\mathrm{d})$. These were selected to show the range of variation in phenotype following sub-MIC antibiotic treatment. Effects on toxin production for all strains and antibiotics are summarized in Table 2. Subinhibitory concentrations of antibiotics tended to delay the growth of the bacteria by increasing the lag period, especially at the highest concentration of antibiotic (1/2 MIC) (strain NCTC 11223 in the presence of clindamycin was an exception to this and is described later). For toxin production, three general consequences were evident: toxin level was increased $(+)$, toxin was produced earlier (E) or toxin level was unaffected or reduced $(-)$.

It should be noted that strain NCTC 11223 is highly resistant to clindamycin (MIC $>512 \mu \mathrm{g} \mathrm{ml}^{-1}$ ) and for this strain only, the sub-MICs are not truly $1 / 2,1 / 4$ and $1 / 8$, but are fractions of $512 \mu \mathrm{g} \mathrm{ml}^{-1}$. When these sub-MIC levels of clindamycin were added, growth of $C$. difficile was not affected (Fig. 2a), but toxin production was affected noticeably. Compared with the antibiotic-free control, toxin was elaborated sooner and reached higher levels than in the absence of clindamycin. Thus, this antibiotic potentiated toxin production by both acceleration and enhancement of production.

Strain 630 grown with amoxycillin is shown in Fig. 2(b). SubMICs resulted in an increased lag period and for $1 / 2$ MIC,

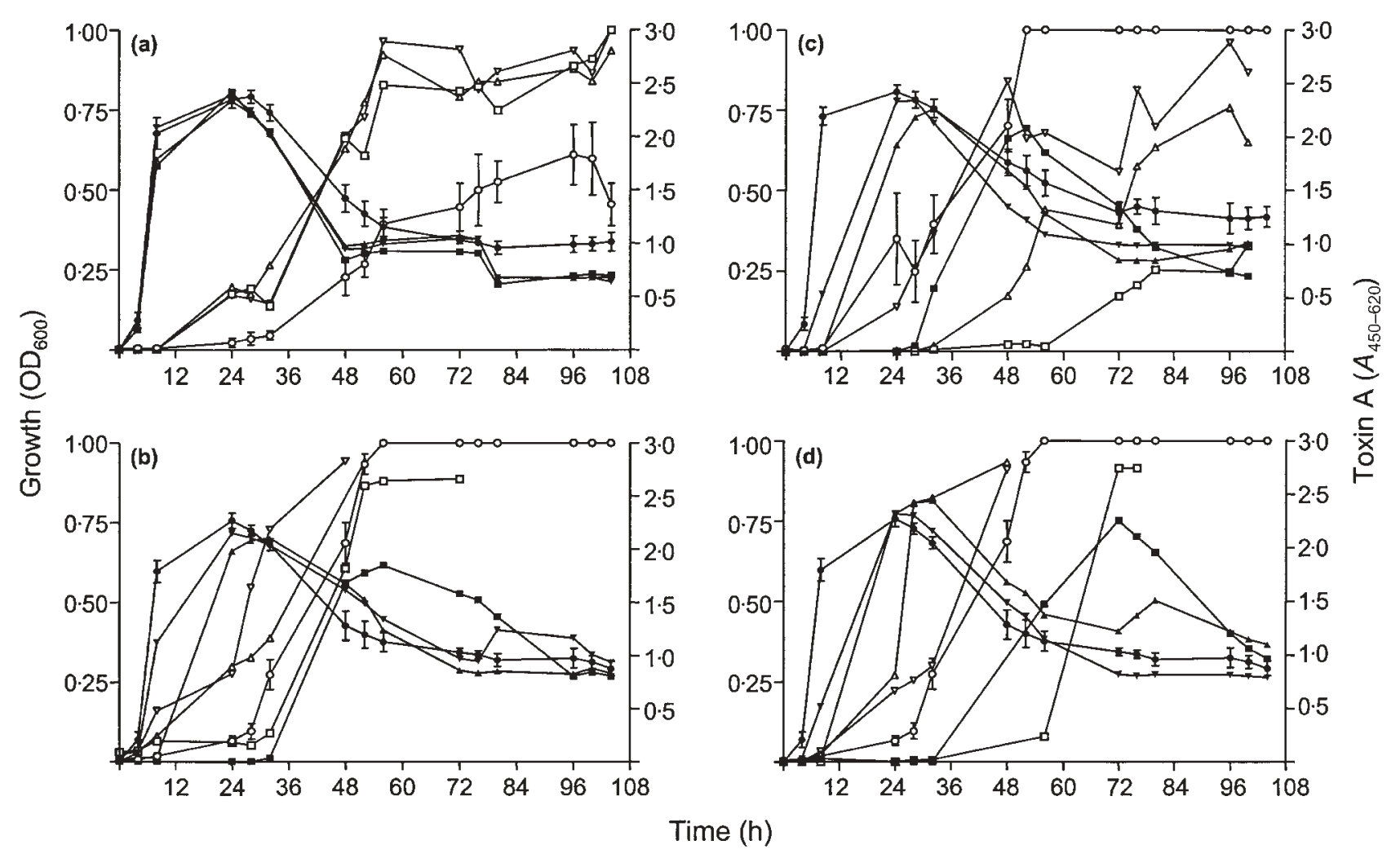

Fig. 2. Growth and toxin production by: (a) strain NCTC 11223 with clindamycin; (b) strain 630 with amoxycillin; (c) strain 338a with cefoxitin; and (d) strain 630 with metronidazole. Controls from Fig. 1 are also shown on these graphs. Closed symbols, growth; open symbols, toxin A. Circles, controls; squares, 1/2 MIC; triangles, 1/4 MIC; inverted triangles, 1/8 MIC. 
Table 2. Summary of effects on toxin production

$1 / 2,1 / 4$ and $1 / 8$ are the concentrations that correspond to $1 / 2,1 / 4$ and 1/8 of the MIC. Abbreviations: Van, vancomycin; Met, metronidazole; Amox, amoxycillin; Clind, clindamycin; Cefo, cefoxitin; Ceft, ceftriaxone; -, no effect; E, elaborated sooner; +, toxin level increased.

\begin{tabular}{|ccccccc|}
\hline Strain & \multicolumn{5}{c|}{ Effect on toxin production } \\
\cline { 2 - 7 } & Van & Met & Amox & Clind & Cefo & Ceft \\
\hline NCTC 11223: & & & & & & \\
$1 / 2$ & - & E & - &,+ E & - & E \\
$1 / 4$ & - & E & E &,+ E & - & - \\
$1 / 8$ &,+ E & E & E &,+ E & - & - \\
$338 \mathrm{a}:$ & & & & & & \\
$1 / 2$ & - & & E & E & - & E \\
$1 / 4$ & - & E & E & E & - & E \\
$1 / 8$ & - & E & - & E & - & - \\
$630:$ & & & & & & \\
$1 / 2$ & E & & E & - & - & - \\
$1 / 4$ & - & E & E & E & - & E \\
$1 / 8$ & - & E & E & E & - & - \\
\hline
\end{tabular}

toxin was produced as soon as growth was measurable, rather than later in the growth cycle. When compared to the control, toxin produced by this strain with $1 / 2$ MIC was elaborated at the same time as by the strain without amoxycillin. This effect was less dramatic with the other two concentrations of amoxycillin. Even with a lag in growth, toxin was produced before the control for $1 / 4$ and $1 / 8 \mathrm{MICs}$ of amoxycillin.

Fig. 2(c) shows the results for strain 338a cultured with cefoxitin; antibiotic appears to inhibit toxin production. The growth rate in $1 / 2$ and $1 / 4$ MICs was lower than that of the control. At 1/2 MIC, there was a lag of approximately $24 \mathrm{~h}$ compared with the control, with toxin levels reaching maximum after $48-72 \mathrm{~h}$.

A final example of the effect of sub-MIC amounts of antibiotic is shown in Fig. 2(d), which depicts strain 630 with metronidazole. This was similar to those for strain $338 \mathrm{a}$ with amoxycillin (results not shown), where toxin appeared to be elaborated before the control, even though growth was delayed.

For $C$. difficile, there is a clear relationship between disease and antibiotic usage, such that antibiotics are often a prerequisite for the disease. Broad-spectrum agents have been shown to particularly predispose to C. difficile infection, through depletion of the patient's normal protective bowel microbiota. The equilibrium of the gut, when disturbed, leaves the patient open to opportunistic infection, possibly via the newfound availability of binding sites and nutrients. Thus, suppression of colonization resistance by antibiotics facilitates colonization and promotes disease. Hence, $C$. difficile is the most common cause of nosocomial antibiotic-associated diarrhoea.
It has been proposed that antibiotics may promote CDAD not solely by modulating commensal micro-organisms, but also by physiological effects that influence pathogenicity (Lorian \& Gemmell, 1994). Several early reports (Onderdonk et al., 1979; Honda et al., 1983) suggested that certain antibiotics potentiated the production of toxins $\mathrm{A}$ and/or $\mathrm{B}$, the main recognized virulence factors of $C$. difficile. Furthermore, antibiotics have been shown to affect the expression of virulence factors in other species, including Escherichia coli, Vibrio cholerae and various staphylococci (Levner et al., 1977; Lorian, 1971; Yoh et al., 1983). Determining the effect of antibiotics on virulence factor expression in an organism for which antibiotics are important triggers of disease is therefore crucial. Our work has focused on the effect of subinhibitory concentrations of six antibiotics, including those that precipitate disease and those used for treatment, on the growth and production of toxin A by three strains of $C$. difficile.

This study has shown clearly that there is heterogeneity between strains with respect to growth, MICs and toxin levels that are produced. A common effect on the bacterial strains in the presence of antibiotics was the slowing of growth in comparison with the controls; they took longer than the controls to reach stationary phase, either at all three sub-MIC concentrations, or just at the higher concentrations, of antibiotics. In addition to slower growth, the bacteria sometimes failed to achieve the OD that the controls reached. This was seen in many cases with strains cultured with cefoxitin. Even with subinhibitory concentrations, it would still be expected that antibiotics would have an effect on bacterial systems, including growth. Strain NCTC 11223 is highly clindamycin-resistant and the growth of this strain was not at all affected in the presence of this antibiotic. An explanation for this may be that this strain is so well-adapted to this agent that it can function and grow as normal. This strain contains the macrolide, lincosamide and streptogramin B resistance determinant (MLS) that contains the ermB gene (encoding an RNA methyltransferase), which makes it resistant to these antibiotics (L. J. Drummond, unpublished data). Strain 630 also carries the ermB gene (Farrow et al., 2001), but it has a slightly lower MIC of $512 \mu \mathrm{g} \mathrm{ml}^{-1}$ and its growth is affected by all three sub-MIC concentrations of clindamycin. The reasons for this are uncertain, but heterogeneity between strains was common during this work.

As can be seen in Table 2 and Fig. 2(a, b and d), sub-MIC concentrations of antibiotics can cause quicker elaboration of toxin A when compared with the antibiotic-free control. Antibiotics, even at sub-MIC concentrations, can be expected to cause stress to the bacteria. Bacteria under stress 'switch on' a catalogue of genes, by which the toxin promoters may be affected. To support this, TcdD - the putative positive regulator of toxin genes - shows similarity to UviA, the UV-inducible regulator from Clostridium botulinum (Mani \& Dupuy, 2001). Onderdonk et al. (1979) showed that the stress of increased temperature led to greater cytotoxin production. In the same paper, the authors demonstrated an increase in toxin in the presence of 
subinhibitory concentrations of vancomycin and penicillin. Karlsson et al. (2003) also showed temperature to be a controlling factor for toxin and TcdD expression. It has been shown by Hennequin et al. (2001) that $C$. difficile cultured in the presence of antibiotics produces greater levels of GroEL, a chaperone from the heat-shock protein 60 (Hsp60) family. These examples all serve to suggest that toxin promoters can respond to multiple environmental stresses. Inducing this stress response may enable $C$. difficile to survive the gut environment, as GroEL functions as a $58 \mathrm{kDa}$ surface adhesin that may help $C$. difficile to colonize recently vacated binding sites left by the depletion of normal gut flora.

Reference strain NCTC 11223 produces lower levels of toxin than the fully sequenced strain (630) and the 'endemic' strain (338a). During the course of the experiments, samples of strain NCTC 11223 rarely exceeded the limits of the ELISA plate reader $(>3)$, whereas the other two strains commonly reached levels $>3$ after approximately 48 h growth. It was desirable to look at the trends of toxin production; this was achievable by comparing the OD values of the antibiotic-free control and the strain in the presence of antibiotics. Differences in toxin production are not well understood, although Spigaglia \& Manstrantonio (2002) demonstrated strains with variants of TcdC, the putative negative regulator of toxin production. No correlation between disease severity and variant $\mathrm{TcdC}$ strains was found, although it is possible that changes in this protein would affect toxin production. For example, they found one allele with a nonsense mutation that reduced the TcdC protein from 232 to 61 aa. Lack of functional protein may lead to abrogated repression of the toxin genes. This may be an explanation for the differences that are common between strains of $C$. difficile. PCR-based analysis would show whether the strains used differed in their tcdC alleles.

In addition to disruption of the barrier flora in C. difficile disease, antibiotics also appear to increase the stress response in the bacteria. For example, upregulation of the adhesin GroEL may increase the virulence of the infecting C. difficile by aiding its utilization of the new niche. The reason for producing toxins in the gut is unclear but, as they are upregulated during glucose starvation, their purpose may be to cause cell disruption for the acquisition of nutrients (Dupuy \& Sonenshein, 1998).

The results presented here show that there is no consistent relationship between antibiotics and growth or toxin production by C. difficile. Antibiotics that are considered to be important for precipitation of the disease appear to have different effects on different strains; this is also true for antibiotics used for treatment, so the relationship appears to be much more complicated than thought previously. The effects of subinhibitory levels of precipitating agents on colonic flora may well allow the overgrowth of $C$. difficile and may also have a significant effect on the rate and/or level of toxins produced once colonization occurs. The impact of this in the patient is unclear, but production of higher levels of toxin earlier in the disease could prove to be detrimental.
Perhaps the most important aspect of this study is the clear demonstration of variation in the response of strains to the same antibiotic. This may have important implications for the virulence potential of different strains.

\section{ACKNOWLEDGEMENTS}

L. J. D. is the holder of an MRC studentship.

\section{REFERENCES}

Barc, M. C., Depitre, C., Corthier, G., Collignon, A., Su, W. J. \& Bourlioux, P. (1992). Effects of antibiotics and other drugs on toxin production in Clostridium difficile in vitro and in vivo. Antimicrob Agents Chemother 36, 1332-1335.

Brown, R., Collee, J. G. \& Poxton, I. R. (1996). In Mackie and McCartney Practical Medical Microbiology, 14th edn, pp. 507-511. Edited by J. G. Collee, A. G. Fraser, B. P. Marmion \& A. Simmons. Edinburgh: Churchill Livingstone.

Dupuy, B. \& Sonenshein, A. L. (1998). Regulated transcription of Clostridium difficile toxin genes. Mol Microbiol 27, 107-120.

Farrell, R. J. \& LaMont, J. T. (2000). Pathogenesis and clinical manifestations of Clostridium difficile diarrhea and colitis. Curr Top Microbiol Immunol 250, 109-125.

Farrow, K. A., Lyras, D. \& Rood, J. I. (2001). Genomic analysis of the erythromycin resistance element Tn5398 from Clostridium difficile. Microbiology 147, 2717-2728.

Hennequin, C., Collignon, A. \& Karjalainen, T. (2001). Analysis of expression of GroEL (Hsp60) of Clostridium difficile in response to stress. Microb Pathog 31, 255-260.

Honda, T., Hernadez, I., Katoh, T. \& Miwatani, T. (1983). Stimulation of enterotoxin production of Clostridium difficile by antibiotics. Lancet $\mathrm{i}$, 655.

Ikeda, D., Karasawa, T., Yamakawa, K., Tanaka, R., Namiki, M. \& Nakamura, S. (1998). Effect of isoleucine on toxin production by Clostridium difficile in a defined medium. Zentbl Bakteriol 287, 375-386.

Karlsson, S., Burman, L. G. \& Åkerlund, T. (1999). Suppression of toxin production in Clostridium difficile VPI 10463 by amino acids. Microbiology 145, 1683-1693.

Karlsson, S., Dupuy, B., Mukherjee, K., Norin, E., Burman, L. G. \& Åkerlund, T. (2003). Expression of Clostridium difficile toxins A and B and their sigma factor TcdD is controlled by temperature. Infect Immun 71, 1784-1793.

Levner, M., Wiener, F. P. \& Rubin, B. A. (1977). Induction of Escherichia coli and Vibrio cholerae enterotoxins by an inhibitor of protein synthesis. Infect Immun 15, 132-137.

Lorian, V. (1971). Effect of antibiotics on staphylococcal hemolysin production. Appl Microbiol 22, 106-109.

Lorian, V. \& Gemmell, C. (1994). Effect of low antibiotic concentrations on ultrastructure, virulence and susceptibility to immunodefences. In Antibiotics and Laboratory Medicine, 3rd edn, pp. 493-555. Edited by V. Lorian. Baltimore: Williams \& Wilkins.

Mani, N. \& Dupuy, B. (2001). Regulation of toxin synthesis in Clostridium difficile by an alternative RNA polymerase sigma factor. Proc Natl Acad Sci U S A 98, 5844-5849.

McCoubrey, J., Starr, J., Martin, H. \& Poxton, I. R. (2003). Clostridium difficile in a geriatric unit: a prospective epidemiological study employing a novel S-layer typing method. J Med Microbiol 52, 573-578.

Mylonakis, E., Ryan, E. T. \& Calderwood, S. B. (2001). Clostridium difficile-associated diarrhea: a review. Arch Intern Med 161, 525-533. 
National Committee for Clinical Laboratory Standards (1997). Methods for Antimicrobial Susceptibility Testing of Anaerobic Bacteria, 4th edn. Approved standard, NCCLS publication no. M11-A4. Villanova, PA: NCCLS.

Onderdonk, A. B., Lowe, B. R. \& Bartlett, J. G. (1979). Effect of environmental stress on Clostridium difficile toxin levels during continuous cultivation. Appl Environ Microbiol 38, 637-641.

Spigaglia, P. \& Mastrantonio, P. (2002). Molecular analysis of the pathogenicity locus and polymorphism in the putative negative regulator of toxin production (TcdC) among Clostridium difficile clinical isolates. J Clin Microbiol 40, 3470-3475.

Yamakawa, K., Karasawa, T., Ikoma, S. \& Nakamura, S. (1996). Enhancement of Clostridium difficile toxin production in biotin-limited conditions. J Med Microbiol 44, 111-114.

Yoh, M., Yamamoto, K., Honda, T., Takeda, Y. \& Miwatani, T. (1983). Effects of lincomycin and tetracycline on production and properties of enterotoxins of enterotoxigenic Escherichia coli. Infect Immun 42, $778-782$. 